Rafidain Journal of Science

Vol. 29, No.2, pp.64 -72, 2020

DOI

https://rsci.mosuljournals.com

ISSN: 1608-9391 e-ISSN: 2664-2786

Received $3 / 2 / 2020$

Accepted 17/5 /2020
استخدام معادلات حالة مختلفة لاراسة نسبة الحجم، معامل المرونة الحجمي وفجوة الطاقة في النانو جرمانيوم تحت الضغط العالي

اسماء فريد عبد اللطيف

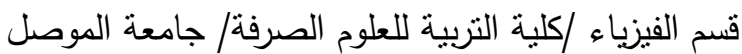

E-mail: violetrose2222@gmail.com

عدنان محمد الثيخ

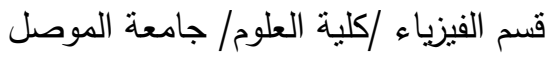

E-mail: dr.adnan@uomosul.edu.iq

الملخص

تم في هذا البحث استخدام معادلات الحالة (معادلة برخ-مرنكهان، معادلة فينيت،

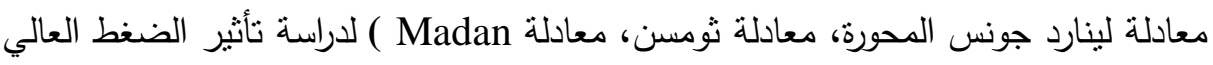

على النانو جرمانيوم من خلال احتساب التغير بالحجم النوعي V/Vo، وتغير معادير معامل المرونة الحجمي B المستخدمة نوافقا مع النتائج التجريبية عند حساب V/V للنانو -جرمانبوم تحت الضغط العالي وزيادة في معامل المرونة الحجمي B مع زيادة الضغط العالي، وزيادة فجوة الطاقة E للنانو جرمانيوم مع زيادة الضغط العالي.

الكلمات الدالة: النانو جرمانيوم، الضغط العالي، معادلات الحالة، فجوة الطاقة، معامل المرونة الحجمي. 


\section{المقدمة}

العلاقة التي تربط الحجم(V) والضغط (P) ودرجة الحرارة (T) لمنظومة معينة، يطلق عليها معادلة الحالة(EOS). وتفيد معادلة الحالة في تحليل الخصائص الحرارية وخصائص المرونة للمنظومة المعنية. والمعروف ان هنالك معادلة حالة عامة للغازات، تعتمد على مفهوم الغاز المثالي. الا ان الامر ليس كذلك في المواد الصلبة اذ ليس هناللك معادلة حالة عامة للمواد الصلبة حتى وقتتا الحاضر .

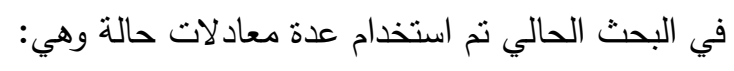

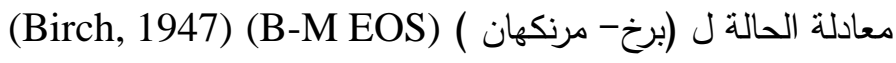

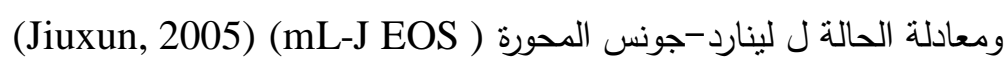

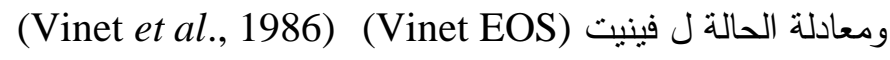
ومعادلة الحالة ل مرنكهان (Murnaghan EOS, 1937) (Muranghan ) ومعادلة الحالة ل ثومسن (Thomsen EOS) (Thom, 1970)

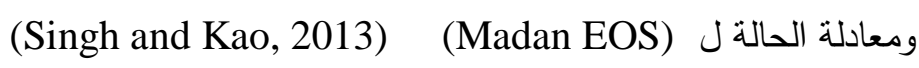
اذ تقع هذه المعادلات المختلفة ضمن اصناف مختلفة لمعادلات الحالة. فمعادلات الحالة تصنف الى ثلاثة اصناف: أ- معادلات حالة تعتمد على تعريف المطاوعة المحددة منل معادلة الحالة لـ ( برخ - مرنكهان ).

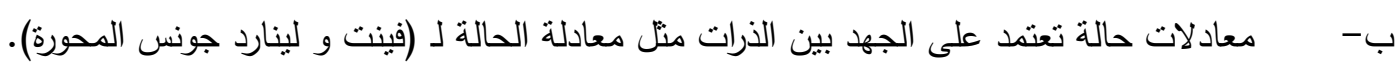

ت- معادلات حالة تعتمد على العلاقة المفترضة بين المتغيرات منل معادلة الحالة لـ ( مرنكهان ). فضلا عن معادلات الحالة التي تعالج المواد العيانية (Bulk materials)، فان عددا من معادلات الحالة التهات التي تعالج مواد النانو

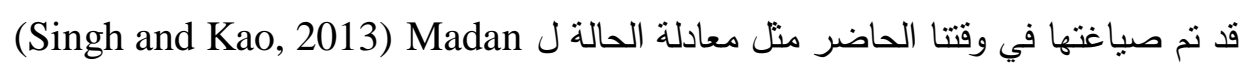
ان الجرمانيوم هو أحد أشهر وابسط المواد شبه الموصلة ويقع ضمن الزمرة الرابعة (IV) من الجدول الدوري. يمنلك

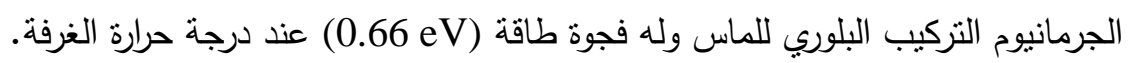
ان وضع تعريف شامل ودقيق للمواد شبه الموصلة ليس بالأمر اليسير خاصة وان التعريف قد ينتاول خاصية معينة من خواص

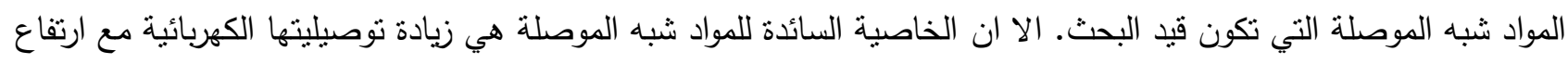
درجة حرارة العينة او بمعنى آخر انخفاض مقاومتها الكهربائية وهذا عكس سلوك المواد الموصلة، رغم ان هذه السمة ليست قائمة لكل مديات درجات الحرارة او لكل المواد شبه الموصلة. تم في هذا البحث استخدام معادلات حالة مختلفة لحساب نغير الحجم النوعي للنانو جرمانيوم وحساب تغير معامل المرونة الحجمي وحساب تغير فجوة الطاقة للجرمانيوم تحت الضغط العالي باستخدام معادلات حالة بمعطيات النانو - جرمانيوم.

\section{التفاصيل النظرية}

معادلات الحالة استخدمت في هذا البحث عدة معادلات حالة وهي:

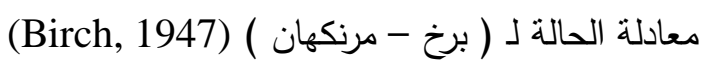

$P_{(B-M)}=\frac{3 B_{0}}{2}\left[\left(\frac{V}{V_{0}}\right)^{-\frac{7}{a}}-\left(\frac{V}{V_{0}}\right)^{-\frac{5}{a}}\right]\left[1+\frac{3}{4}\left(B_{0}^{\prime}-4\right)\left(\left(\frac{V}{V_{0}}\right)^{-\frac{2}{a}}-1\right)\right]$ ويعبر عنها بالصيغة: 


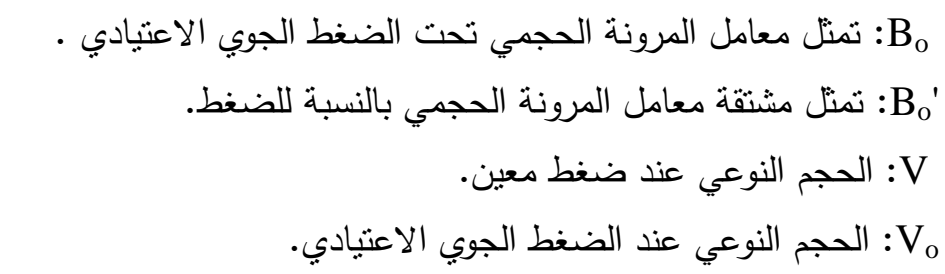

معادلة الحالة ل ( لينارد -جونس ) المحورة (Jiuxun, 2005)

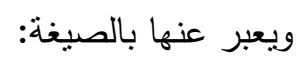

$$
\begin{array}{r}
P_{L-D)}=\frac{B_{0}}{n}\left(\frac{V o}{V}\right)^{n}\left[\left(\frac{V o}{V}\right)^{n}-1\right] \\
\mathrm{n}=\frac{1}{3} \mathrm{~B}_{\mathrm{o}}{ }^{\prime}
\end{array}
$$

معادلة الحالة ل فينيت (Vinet et al., 1986) (Vinet EOS)

ويعبر عنها بالصيغة التالية:

$P_{\text {vinet }}=3 B_{0}\left(\frac{V}{V_{0}}\right)^{-\frac{a}{a}}\left(1-\left(\frac{V}{V_{0}}\right)^{\frac{1}{a}}\right) \exp \left[\left\{\frac{3}{2}\left(B_{0}^{\prime}-1\right)\right\}\left\{1-\left(\frac{V}{V_{0}}\right)^{\frac{1}{a}}\right\}\right]$

معادلة الحالة ل ثومسن (Thomsen EOS) (Thom, 1970)

ويعبر عنها بالصيغة التالية:

$P_{T h}=\frac{3 B_{0}}{2}\left(\left(\frac{V}{V_{0}}\right)^{-\frac{1}{a}}-\left(\frac{V}{V_{0}}\right)^{\frac{1}{a}}\right)\left[1+\frac{3}{4} B_{0}^{\prime}\left(1-\left(\frac{V}{V_{0}}\right)^{\frac{2}{a}}\right)\right]$

(Vingh and Kao, 2013) (Madan ( معادلة الحالة ل (v) ويعبر عنها بالصيغة التالية:

$P_{M}=B_{0}\left(1-\left(\frac{V}{V_{0}}\right)\right)+\frac{B_{0}\left(B_{0}^{\prime}+1\right)}{2}\left(1-\left(\frac{V}{V_{0}}\right)\right)^{2}$

B معامل المرونة الحجمي يعرف معامل المرونة الحجمي بانه مقياس لمقاومة المادة للانضغاط عند تسليط ضغط خارجي على تللك المادة. وهو

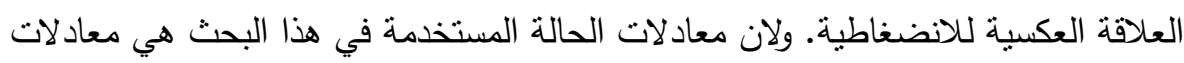

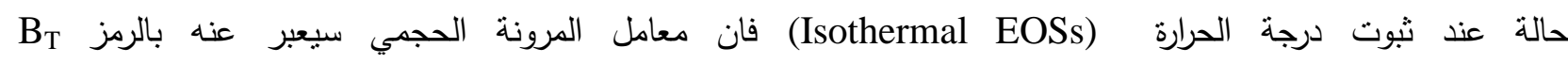
(Isothermal Bulk modulus)

$$
B_{T}=-V \frac{d p}{d v}
$$

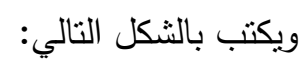

اذ ان BT: تمنل معامل المرونة الحجمي عند ثبوت درجة الحرارة. معامل المرونة الحجمي تحت الضغط العالي ان تغير معامل المرونة الحجمي تحت الضغط العالي قد تمت دراسته من قبل العديد من الباحثين (Boring, Lei, and Cui 1986; Chandra et al., 2016; Pandey et al., 2012; Sushil et al., 2004) 
نظرا لدوره الاساسي في معادلات الحالة وفي توصيف سلوك المواد الصلبة تحت الضغط العالي.

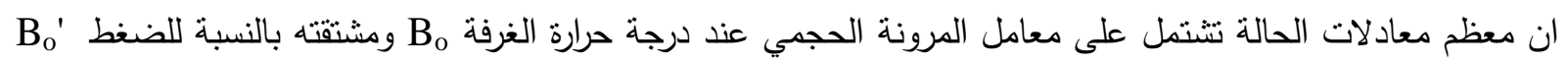
كمعطيات اولية لأجراء الحسابات. ان هذه المعطيات عادة تستخدم في معادلات الحالة لحسابات تغير الحجم ومعامل المرونة

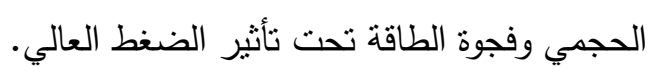
تم في هذا البحث صياغة تغير معامل المرونة الحجمي تحت نأثير الضغط العالي باستخدام معادلات الحالة المختلفة: i

$$
\begin{gathered}
\left(\frac{d p}{d v}\right)_{B-M}=\frac{3 B_{0}}{2}\left[-\frac{7}{3}\left(\frac{V^{\frac{7}{a}-1}}{V_{0}^{-\frac{7}{3}}}\right)+\frac{5}{3}\left(\frac{V^{\frac{5}{3}-1}}{V_{0}^{-\frac{5}{a}}}\right)+\frac{9}{4}\left(B_{0}^{\prime}-4\right) *\left(\frac{V^{-1-1}}{V_{0}^{-1}}\right)-\frac{7}{2} *\right. \\
\left.\left(B_{0}^{\prime}-4\right)\left(\frac{V^{\frac{7}{3}-1}}{V_{0}^{-\frac{7}{8}}}\right)+\frac{5}{4}\left(B_{0}^{\prime}-4\right)\left(\frac{V^{\frac{5}{3}-1}}{V_{0}^{-\frac{5}{3}}}\right)\right]
\end{gathered}
$$
فمن معادلة (1) تم حساب

بتعويض معادلة (7) بالمعادلة (6) نحصل على: $B_{T_{(B-M)}}=\frac{3 B_{0}}{2}\left[\frac{7}{3}\left(\frac{V}{V_{0}}\right)^{-\frac{7}{a}}-\frac{5}{3}\left(\frac{V}{V_{0}}\right)^{-\frac{5}{a}}-\frac{9}{4}\left(B_{0}^{\prime}-4\right)\left(\frac{V}{V_{0}}\right)^{-3}+\frac{7}{2}\left(B_{0}^{\prime}-4\right)\left(\frac{V}{V_{0}}\right)^{-\frac{7}{a}}-\frac{5}{4}\left(B_{0}^{\prime}-\right.\right.$ 4) $\left.\left(\frac{V}{V_{0}}\right)^{-\frac{5}{a}}\right]$

وبنفس الطريقة تم حساب

$$
B_{T_{(m L-I)}}=B o\left(\frac{V o}{V}\right)^{n}\left[2\left(\frac{V o}{V}\right)^{n}-1\right]
$$

تغير معامل المرونة (معادلة لينارد جونس - المحورة) (ii)

(iii)

$B_{T \text { (vinet) }}=\left[2 B_{o}\left(\frac{V}{V_{0}}\right)^{-\frac{a}{a}}-\left(\frac{V}{V_{o}}\right)^{-\frac{1}{a}}+B o\left(\frac{V}{V_{o}}\right)^{-\frac{1}{a}}+\frac{3}{2} B o\left(B o^{\prime}-1\right)\left(\left(\frac{V}{V_{o}}\right)^{-\frac{1}{a}}-\right.\right.$

$$
\text { 1) }] \exp \left[\left\{\frac{3}{2}\left(B o^{s}-1\right)\right\}\left(1-\frac{V}{V_{o}}\right)^{\frac{1}{3}}\right]
$$

تغير معامل المرونة (معادلة ثومسن) (iv)

$$
B_{T(T H)=}\left(\frac{B o}{2}\right)\left[\left(\frac{V}{V o}\right)^{-\frac{1}{a}}+\frac{3}{4}\left(B o^{\prime}\left(\frac{V}{V o}\right)^{-\frac{1}{a}}+\frac{3}{2} B o^{\prime}\left(\frac{V}{V o}\right)^{\frac{1}{a}}+\left(\frac{V}{V}\right)^{\frac{1}{a}}-\frac{9}{4} B o^{\prime}\left(\frac{V}{V o}\right)\right)\right]
$$

(Madan تغير معامل المرونة ( معادلة (v)

$$
\mathrm{B}_{\mathrm{T}(\text { Madan })}=\left(\frac{V}{V o}\right) B o+\left(\frac{V}{V o}\right)\left(1-\frac{V}{V o}\right)\left(\frac{B o\left(B o^{r}+1\right)}{2}\right)
$$


ظاهرة شبه التوصيل في الجرمانيوم

ان منشأ ظاهرة شبه التوصيل في الجرمانيوم النقي يمكن توضيحها باستخدام نموذج الجمع الخطي للمدارات الذرية

(Linear combination of atomic orbitals -(LCAO)) فذرة الجرمانيوم المعزولة تمنلك عدد ذري 32 (Hameed and AL-Sheikh, 2011) وترتيب الكتروني يأخذ الصيغة: $1 \mathrm{~s}^{2} 2 \mathrm{~s}^{2} 2 \mathrm{p}^{6} 3 \mathrm{~s}^{2} 3 \mathrm{p}^{6} 3 \mathrm{~d}^{10} 4 \mathrm{~s}^{2} 4 \mathrm{p}^{2}$

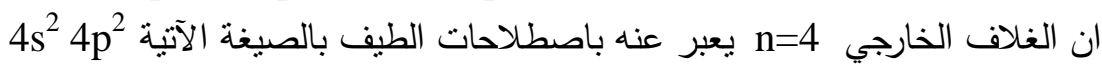

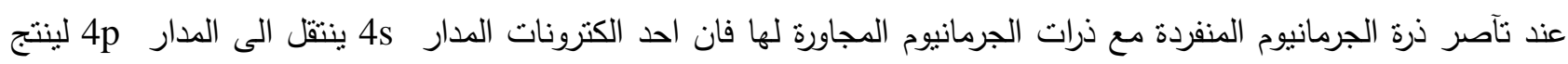
الترتيب الالكتروني، ان هذه المدارات المهجنة نولد مدارات متجهة نحو زوايا رباعي الاوجه المنتظم ( regular tetrahedron )،

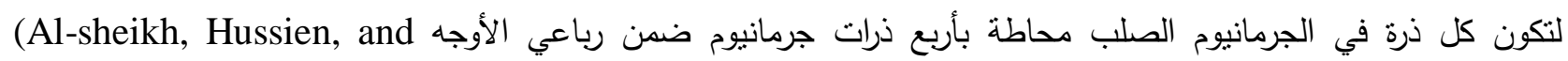
.Abdullah, 2019)

ان اشباه الموصلات في البعد الواحد او البعدين قد اظهرت خصائص الكترونية وضوئية تختلف حسب حجم الجسيمات وهذا ما جعل هذا البحث يتتاول دراسة النانو -جرمانيوم تحت الضغط العالي. اذ تتاول البحث دراسة الخصائص الحرارية وخصائص المرونة وفجوة الطاقة تحت الضغط العالي مقارنة مع البيانات التجريبية المتاحة(Wang et al., 2005).

تغير فجوة الطاقة تحت الضغط العالي ودرجات الحرارة المختلفة

يعبر عن تغير فجوة الطاقة للجرمانيوم تحت الضغط العالي وعند درجات حرارة مختلفة بالعلاقة: $E_{g}(P, T)=E_{g}(0)+\alpha P-\frac{K T^{2}}{T+C} \quad$ (Rodríguez et al., 2009)

اذ ان K و C معا معاملات درجة الحرارة

• (pressure coefficient $d E_{g} / d p$ ) معاجه $\alpha$

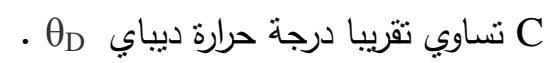

$\mathrm{E}_{\mathrm{g}}(\mathrm{T})=\mathrm{E}_{\mathrm{g}}(0)-\frac{\mathrm{KT}^{2}}{\mathrm{~T}+\mathrm{C}}$

$\mathrm{E}_{\mathrm{g}}(\mathrm{p})=\mathrm{E}_{\mathrm{g}}(0)+\alpha \mathrm{P}$

وعند تعويض T=0 في المعادلة (13) فان المعادلة تؤول للصيغة (15) كالآتي فعندما يكون الضغط المسلط هو الضغط الجوي الاعتيادي p=0 تصبح المعادي درئة بالثكل التالي

\section{الحسابات وإلنتائج}

حساب تغير V/Vo للنانو جرمانيوم باستخدام معادلات حالة مختلفة

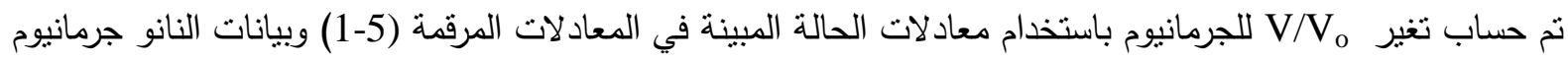
الدونة في (الجدول 1) وحصلنا على النتائج المبينة في الثكل (1) مقارنة مع النتائج التجريبية (Wang et al., 2005) الجدول 1: قيم معامل المرونة الحجمي ومشتقة معامل المرونة الحجمي للنانو جرمانيوم وللجرمانيوم العياني

\begin{tabular}{|c|c|c|r|}
\hline Material & Bo & Bo' $^{\prime}$ & Ref. \\
\hline Bulk $(\mathrm{Ge})$ & 72 & 4.31 & (Tripathi et al., 2006) \\
\hline Nano $(\mathrm{Ge})$ & 92 & 4 & (Wang et al., 2005) \\
\hline
\end{tabular}




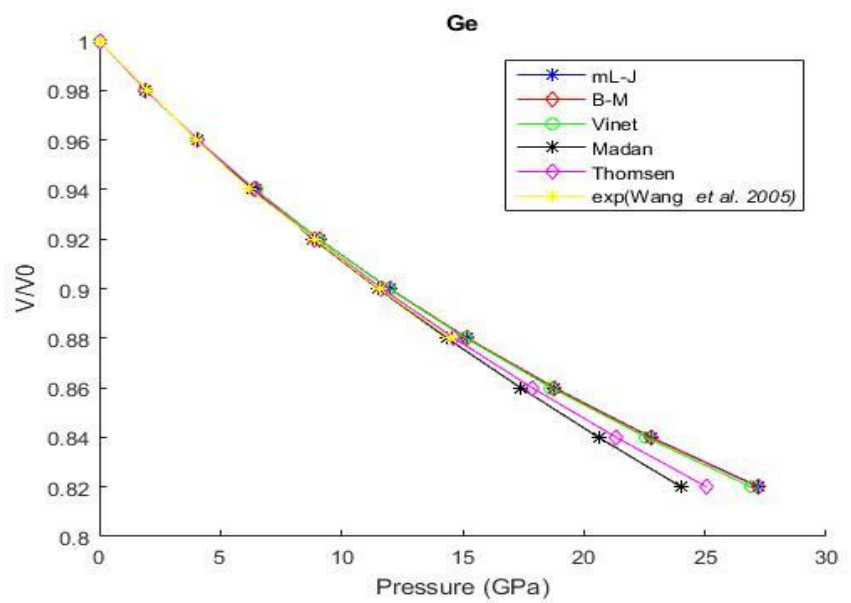

الثكل 1: تغير V/Vo للنانو جرمانيوم تحت تأثير قيم مختلفة للضغط العالي باستخدام معادلات حالة مختلفة حساب تغير معامل المرونة الحجمي للنانو جرمانيوم باستخدام معادلات الحالية

تم استخدام معادلات تغير معامل المرونة الحجمي مع الضغط المبينة بالمعادلات المرقمة (12-8) واثشير لها مرة اخرى في الفقرة(2-3)، وحصلنا على النتائج المبينة في الشكل (2). اذ تظهر جميع النتائج المستتدة لمعادلات الحالة المختلفة زيادة قيم معامل المرونة الحجمي تحت تأثثر الضغط العالي، رغم تقارب نسبة الزيادة بين المعادلات المختلفة، اذ اظهرت معادئ معادلة تغير

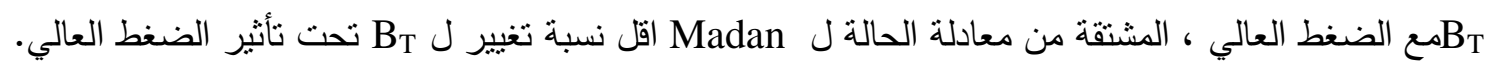

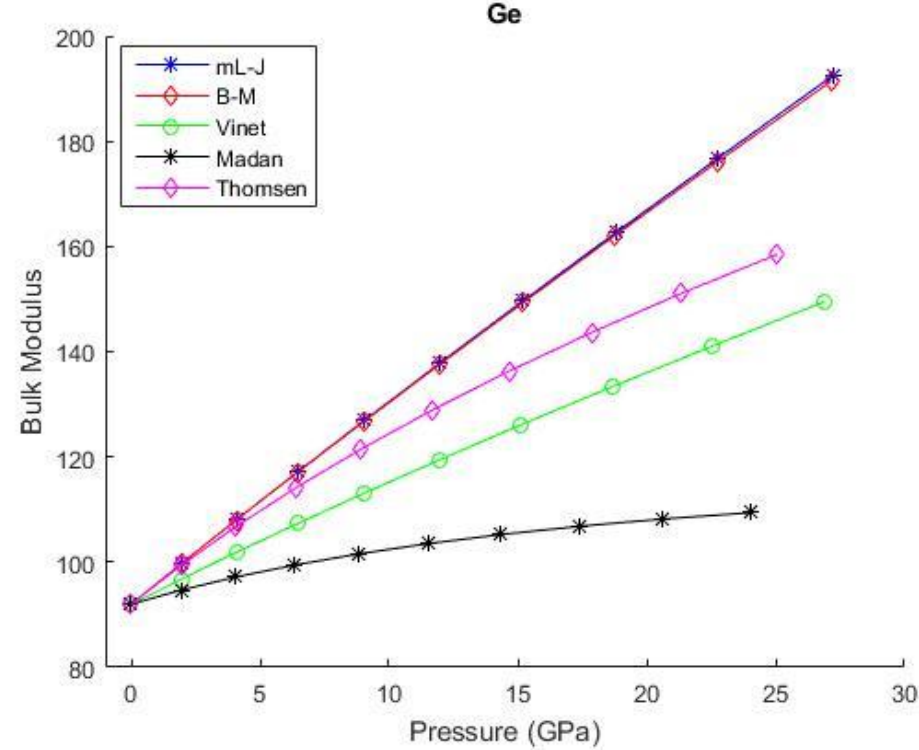

الشكل 2: تغير معامل الحجمي للنانو جرمانيوم تحت تأثير الضغط العالي باستخدام معادلات حالة مختلفة

حساب تغير فجوة الطاقة الجدول (2) يوضح قيم المعاملات الخاصة بالنانو - جرمانيوم 


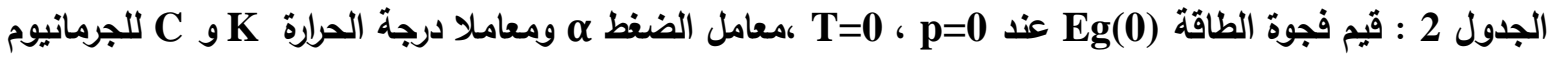
(Levinshtein, Rumyantsev, and Shur, 2001)

\begin{tabular}{|c|c|c|c|}
\hline $\mathbf{E}_{\mathrm{g}}(\mathbf{0}) \mathbf{e V}$ & $\boldsymbol{\alpha} * \mathbf{1 0}^{-\mathbf{3}} \mathbf{e V} / \mathbf{k b a r}$ & $\mathbf{K}^{*} \mathbf{1 0} \mathbf{0}^{-\mathbf{4}} \mathbf{e V} / \mathbf{K}$ & $\mathbf{C ~ K}$ \\
\hline 0.742 & 5.1 & 4.8 & 235 \\
\hline
\end{tabular}

بتعويض قيم معاملات النانو -جرمانيوم من (الجدول 2) بالمعادلة (15) نم حساب تغير فجوة الطاقة تحت تأثير الضغط العالي اذ اظهرت النتائج زيادة فجوة الطاقة للجرمانيوم مع زيادة الضغط المسلط على العينة كما موضح في الثكل (3) (تمت الحسابات بمعادلات الحالة المبينة في المعادلات 1 الى 5 لتغير الحجم النوعي الى 12 وحساب تغير فجوة الطاقة باستخدام المعادلة 15 واستخدمت برامج حاسوبية مكتوبة من قبلنا لبرمجة معادلات الحالة

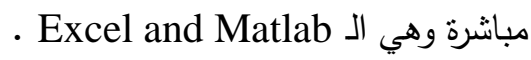

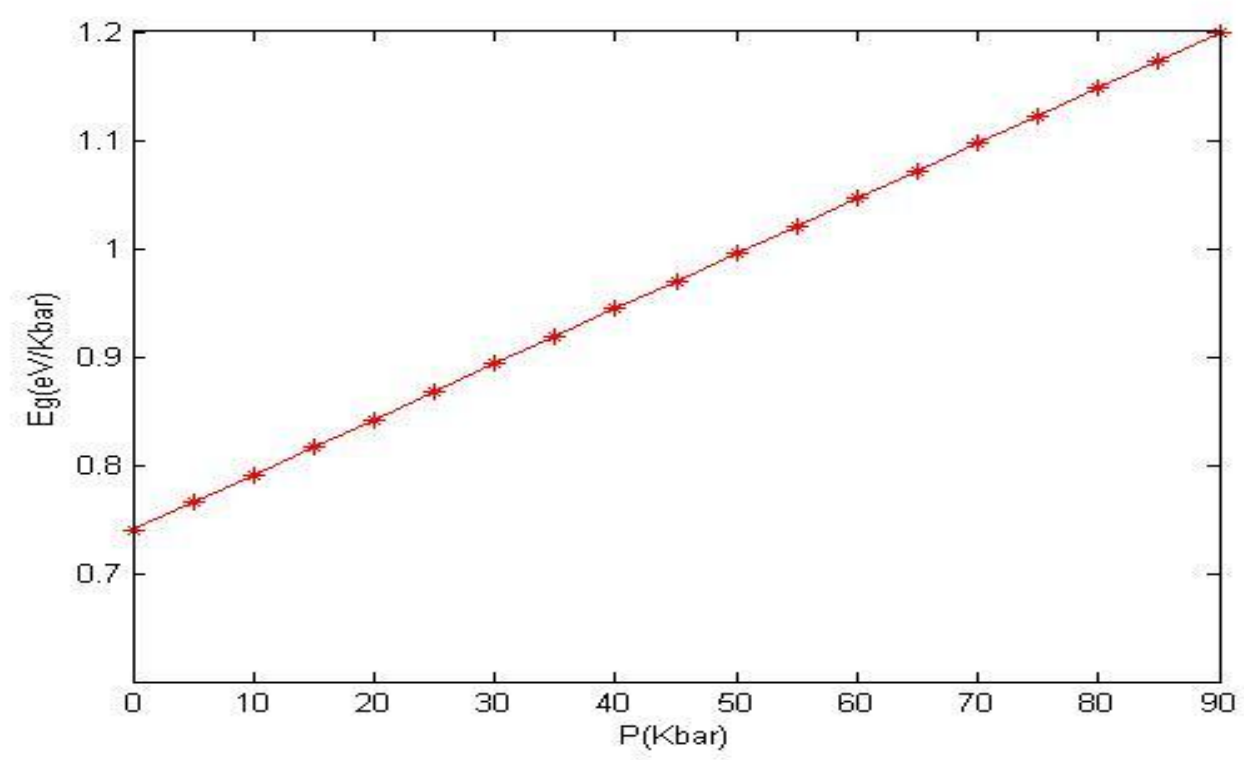

الثكل 3: تغير فجوة الطاقة Eg للنانو جرمانيوم تحت تأثير الضغط العالي باستخدام المعادلة (15)

\section{المناقشة}

1- ان قيمة معامل المرونة الحجمي للنانو جرمانيوم اكبر من معامل المرونة الحجمي للجرمانيوم العياني كما مبين في الفقرة

$$
\text { . (3-1) من البند } 3 \text { (1) }
$$

من الثكل (1) نلاحظ انه رغم نوافق جميع معادلات الحالة المستخدمة مع النتائج التجريبية لتغير V/V تحت الضغط

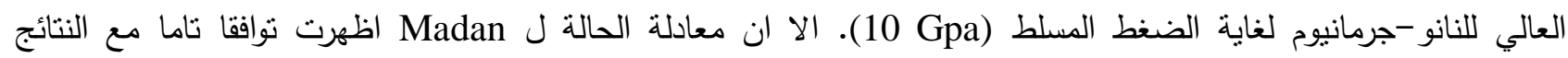
التجريبية اكثر من بقية المعادلات في الضغوط الاعلى من (10Gpa).

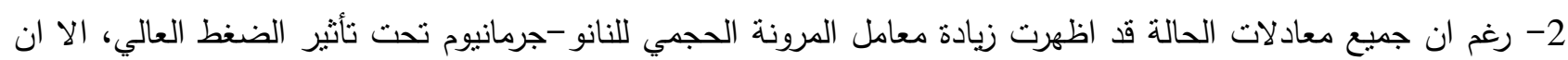
معادلة Madan والتي هي من المعادلات الخاصة بمواد النانو خلافا، لمعادلات (لينارد جونس المحورة، برخ مرنكهان، فينيت، ثومسن) اللواتي يعالجن المواد العيانية ونم استخدامها في حسابات النانو لغرض المقارنة، اظهرت معادلة Madan نسبة زيادة في معامل المرونة الحجمي تحت الضغط العالي اقل من بقية المعادلات كما مبين في الثكل (2). 


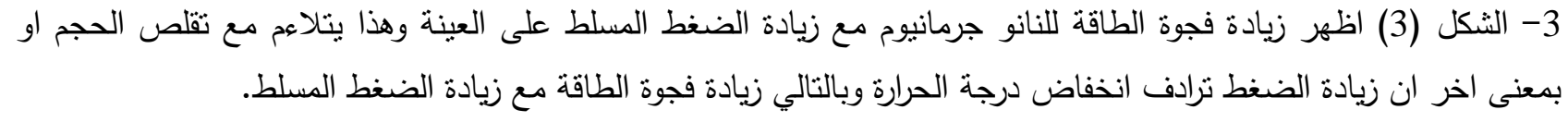

Al-sheikh, A. M.; Mumtaz, M. H.; Siham, J. A. (2019). Pressure and temperature dependence of energy gap in sic and si1-xgex. Raf. J. Sci., 28(4),53-61.

Birch, F. (1947). Finite elastic strain of cubic crystals. Physical Review. 71(11), 809-24.

Boring, N.J.M.; Lei, X. L.; Cui, H. L. (1986). Dielectric interpretation of lei-ting nonlinear forcemomentum - balance transport equation for isotheriiial resistivity. Physical Review B 33(10), 6929-35.

Chandra Usha, K. K.; Pandey, G. P.; Surinder, M. S. (2016). High-Pressure investigations on Piplia Kalan Eucrite Meteorite using in-Situ X-Ray diffraction and 57Fe Mössbauer Spectroscopic Technique up to $16 \mathrm{GPa}$. Geoscience Frontiers . 7(2), 265-71.

Hameed, Kh.S. ; AL-Sheikh, A.M. (2011). Theoretical study of energy gap for silicon and germanium under high pressure. Raf. J. Sci., 22(2), 58-68.

Jiuxun, S. (2005). A modified lennard-jones-type equation of state for solids strictly satisfying the spinodal condition. J. Physics Condensed Matter. 17(12).

Levinshtein, M. E.; Sergey, L. R.; Michael, S. S. (2001). Properties of advanced semiconductor materials: GaN, AIN, InN, BN, SiC, SiGe .

Murnaghan, FD. (1937). Finite deformations of an elastic solid. American J. Mathematics.

Pandey, K. K.; Nandini Garg, A. K.; Mishra; Surinder, M. S. (2012). High pressure phase transition in Nd2O3. J. Physics: Conference Series. 377(1), 2-6.

Rodríguez, A.H.; Trallero-Giner, C. C.; Duque, A.; Vázquez, G. J. (2009). Optical transition in self-assembled inas/gaas quantum lens under high hydrostatic pressure. J. Appl. Physics $105,044308$.

Singh, M.; Moruti K. (2013). Study of nanomaterials under high pressure. Advances in Nanoparticles. 02(04), 350-57.

Sushil, K.; Arunesh, K.; Singh, P. K.; Sharma, B. S. (2004). Analysis of finite-strain equations of state for solids under high pressures. Physica B: Condensed Matter 352(1-4), 134-46.

Thomsen, L. (1970). On the fourth-order anharmonic equation of state of solids. J. Physics and Chemistry of Solids. 31(9), 2003-16.

Tripathi, S. K.; Anup Thakur, J.; Sharma, G.; Singh, V.; Sharma, K. P.; Singh, G. S.; Saini, S.; Goyal, N. (2006). Proton-Induced changes on the optical parameters of a(Ge20Se80)0.96Ag0.04 Thin Film. J. Mater. Sci., 41, 1847-50.

Vinet, P.; Ferrantef, J.; Smith, J. R.; Rose, J. H. (1986). A universal equation of state for solids. J. Physics C: Solid State Physics. 19(20), L467-73. 
Wang, H., Wang, Y.; Chen, W.; Liu, J. F.; Jiang, J. Z.; Olsen, J. S.; Leif Gerward ; Gleiter, H. (2005). High-Pressure structural behaviour of nanocrystalline Ge. in Joint 20th AIRAPT 43rd EHPRG International Conference of High Pressure Sci. and Technol., 19(15).

\title{
Using Different Equations of States to Study Volume Ratio, Bulk Modulus and Energy Gap in Nano Ge Under High Pressure
}

\author{
Asmaa F. Abdulateef \\ Department of Physics/ College of Education for Pure Science/ University of Mosul \\ Adnan M. Al-Shiekh \\ Department of Physics/College of Science/ University of Mosul
}

\begin{abstract}
This research has been used EOSs (Birch-Muranghan, Vinet, mL-J, Thomsen and Madan) to study the effect of high pressure on nano-Ge parameters by calculating compressed volume V/Vo, Bulk modulus BT, Energy gap Eg, under high pressure. All equations used showed compatibility with the experimental results for calculating $\mathrm{V} / \mathrm{Vo}$ of nano-Ge under high pressure, an increase in the Bulk modulus BT with an increase in high pressure and an increase in the energy gap of nano Ge with high pressure.
\end{abstract}

Keywords: Nano-Ge, High pressure, Equations of state, Energy gap, Bulk modulus. 